\title{
A Flaming Heart: Immunotherapy-mediated Myopericarditis
}

\author{
Jingjing Chen ${ }^{1}$, Annya Suman ${ }^{1}$, and Christopher Haas ${ }^{2}$ \\ ${ }^{1}$ MedStar Union Memorial Hospital \\ ${ }^{2}$ MedStar Franklin Square Medical Center
}

July 22, 2020

\begin{abstract}
We report a case of immunotherapy associated myopericarditis in a patient treated with Nivolumab/Ipilimumab. We furthermore performed a comprehensive literature review regarding the biological mechanisms of cancer immunity, the pathophysiology behind immunotherapy related adverse effects (irAEs), current guideline, and the role of immune checkpoint inhibitors to the recurrent irAEs.
\end{abstract}

Journal: Clinical Case Report

Title: A Flaming Heart: Immunotherapy-mediated Myopericarditis

Running title: Immunotherapy-mediated Myopericarditis

Authors:

Jingjing Chen, MD $\mathrm{MPH}^{1^{*}}$; Annya Suman, $\mathrm{MD}^{1^{*}}$; Christopher James Haas, MD $\mathrm{PhD}^{2,3}$

${ }^{1}$ Internal Medicine Resident, MedStar Health Internal Medicine Residency Program, Baltimore, MD

${ }^{2}$ Faculty Physician, MedStar Health Internal Medicine Residency Program, Baltimore, MD

${ }^{3}$ Assistant Professor of Medicine, Georgetown University School of Medicine, Washington, D.C.

${ }^{*}$ Co-first Authors

Manuscript word count: 3554 words (including titles)

Author emails:

- Jingjing Chen (jingjing.chen@medstar.net)

- Annya Suman (annya.suman@medstar.net)

- Christopher James Haas (christopher.j.haas@medstar.net)

\section{Corresponding author contact information :}

Jingjing Chen

Affiliation: Internal Medicine Resident, MedStar Health Internal Medicine Residency Program, Baltimore, MD

201 E. University Pkwy Ste 405 Baltimore, MD 21218-2895

Email: jingjing.chen@medstar.net

Phone number: 443-455-0619 


\section{Introduction}

Immune checkpoint inhibitors have proven to be a promising and novel therapy for a variety of previously refractory malignancies, designed to subvert the cancer-mediated suppression of the normal immune system. Malignancies can adapt to the host environment, exacting a number of signaling cascades to evade host detection. Malignant cells can limit recognition by downregulation of the major histocompatibility complex (MHC) Class I molecules involved in antigen presentation to T-lymphocytes and modulate the tumor microenvironment by dampening regulatory T-lymphocytes (Treg), myeloid-derived suppressor cells, and tumor-associated macrophages via elaboration of multiple immunosuppressive soluble cytokines (TGF- $B$, IL10). ${ }^{1-5}$ Additionally, malignant cells suppress host immune activation by upregulation of specific cell-surface proteins such as Programmed Death Ligand 1 (PD-L1) and Cytotoxic T-lymphocyte-associated protein 4 (CTLA4) - whose ultimate function is to bind to receptors on those inflammatory, tumor-killing, tumorinfiltrating T-lymphocytes and block pathways associated with T-lymphocyte activation, proliferation, in turn limiting malignant cell detection and killing. ${ }^{6-8}$ While many factors play a role in mediating effective immune responses to cancer, T-lymphocytes are a cornerstone of the anti-cancer immune response and are likely a critical factor in determining clinical outcome.

T-lymphocytes target tumor cells mainly by two mechanisms. The first mechanism involves an antigenspecific signal through T-cell receptors (TCRs); the second mechanism involves antigen-nonspecific signals mediated by co-signaling receptors: co-stimulatory or co-inhibitory, which act by accentuating or attenuating T-lymphocyte responses, respectively. ${ }^{9,10}$ CD28 is an important co-stimulatory receptor, whereas CTLA-4 and PD-1 are co-inhibitors. These latter signals are essential to restrict cytotoxic T-lymphocyte effector function. Malignancy-mediated upregulation of PD-L1 and CTLA4 leads to T-lymphocyte anergy and suppression, ultimately leading to evasion of the host immune response. It was subsequently hypothesized that blockade of the PD-1/ PD-L1 interaction or inhibition of the malignancy-mediated upregulation of PD-L1 and CTLA4, would lead to enhanced recognition of malignant cells. Backed by strong pre-clinical evidence, these novel therapies have proven to be very effective in advanced melanoma in phase I-III trials. ${ }^{11-13}$ Two such therapeutics, Nivolumab and Ipilimumab, designed to inhibit PD-1 and CTLA-4, respectively, have steadily expanded their application from advanced melanoma to a variety of other advanced malignancies, including renal cell carcinoma, colorectal cancer, non-small-cell lung cancer. ${ }^{12-18}$

While these immunotherapies have demonstrated enhanced tumor recognition and T-lymphocyte activation, their use has been associated with several deleterious side effects, termed immunotherapy-related adverse events (irAEs). Though the exact pathogenesis of irAEs remains to be fully delineated, it is thought to be driven by the same immunologic mechanisms responsible for the drugs' therapeutic effects, namely blockade of inhibitory mechanisms that suppress the immune system and protect body tissues from an unchecked acute or chronic immune response. ${ }^{19}$ Though a variety of irAEs have been described in the literature colitis, thyroid dysfunction, pneumonitis, hepatitis, and nephritis - cardiac involvement has been noted to be exceedingly rare, with an incidence rate of less than $1 \%$, and commonly manifests as non-specific symptoms - dyspnea, palpitation, fatigue, and myalgia. ${ }^{20-24}$ Here we report a case of dual-immunotherapy associated myopericarditis in an individual with metastatic renal cell carcinoma treated with combinatorial Nivolumab/ Ipilimumab therapy and provide a brief literature review regarding the biological mechanisms of immunotherapy and the pathophysiology behind irAEs.

\section{Case Presentation}

A 63-year-old male with a past medical history notable for a remote, seven pack-year tobacco history and recently diagnosed Stage 4 renal cell carcinoma (PAX 8(+)/TTF-1(-)) complicated by metastasis to the right hilum status post radiation and recent initiation of Nivolumab and Ipilimumab presented to our facility in the context of a two-week history of shortness of breath and fatigue. Of note, four months prior to presentation the patient visited his primary care physician in the context of a three-week history of an unexplained cough. Chest radiograph demonstrated a right hilar mass, which was confirmed on computed tomography, measuring $6.9 \mathrm{~cm} \mathrm{x} 3.3 \mathrm{~cm} \times 4.3 \mathrm{~cm}$, and resulted in severe narrowing of the Superior Vena Cava with associated deformity of the right upper lobe and pulmonary artery. CT also demonstrated the presence of a 
heterogenous left renal mass measuring $6.3 \mathrm{~cm}$ x $5.8 \mathrm{~cm} \times 5.6 \mathrm{~cm}$ and the patient was referred for biopsy which demonstrated metastatic renal cell carcinoma. He was subsequently initiated on radiation therapy directed toward the hilar mass (4000cGY) with a planned two-week course, which he completed 3 weeks prior to our hospital presentation. Following induction of radiation therapy, he was initiated on immunotherapy with Nivolumab/ Ipilimumab. Three days following his second cycle of Nivolumab/ Ipilimumab, he presented to our facility.

On presentation the patient reported shortness of breath that began two days after his first immunotherapy treatment, characterized initially as dyspnea on exertion and fatigue that acutely worsened and progressed following his second cycle of immunotherapy to involve dyspnea at rest. He had no associated fevers, chills, chest pain, cough, leg swelling, recent travel, or sick contacts. On presentation the patient was afebrile, tachycardic (140 beats per minute), and normotensive (SBP ranging from 100-120 $\mathrm{mmHg}$ ), with a preserved oxygen saturation $(97 \%)$ on room air. Initial laboratory workup demonstrated a preserved VBG $(\mathrm{pH}$ 7.39/pCO2 $42.9 \mathrm{~mm} \mathrm{Hg} / \mathrm{pO} 231.5 \mathrm{~mm} \mathrm{Hg} / \mathrm{HCO} 326.2 \mathrm{mEq} / \mathrm{L})$ and NT-proBNP (37 pg/mL), with an elevated troponin of $2.1 \mathrm{ng} / \mathrm{mL}$, and D-dimer $(1.07 \mathrm{mcg} / \mathrm{mL})$. Complete blood count and basic metabolic panel were unremarkable, however aspartate aminotransferase (AST) and alanine aminotransferase (ALT) were elevated to 166 units/L and 129 units/L, respectively. Alkaline phosphatase and bilirubin were within normal limits. Creatine Kinase was elevated to 1266 units/L. Chest computed tomography (CT) demonstrated a $6.7 \mathrm{~cm} \mathrm{x} 4 \mathrm{~cm}$ right hilar mass consistent with prior imaging with no appreciable change in size compared to the chest CT completed four months prior, with continued narrowing of the SVC (Figure 1 ). There were multiple, small scattered irregular nodular densities throughout the right lung suspicious for metastatic disease, without associated pleural effusions. The heterogeneous left-sided renal cell mass measuring $4.6 \mathrm{~cm}$ x $5.3 \mathrm{~cm}$ (Figure 2 ) was once again appreciated, with noted interval decrease in size. Electrocardiogram demonstrated sinus tachycardia, left atrial enlargement, delayed R-wave progression, and subtle, diffuse ST elevations and PR depressions (Figure 3 ). Nuclear medicine scan was performed which demonstrated no evidence of pulmonary embolism.

Due to concern for SVC compression and an associated demand ischemia, he received three liters of crystalloid and was transferred to the medicine service for further evaluation. On presentation to the medicine ward, he remained persistently tachycardic and demonstrated no resolution of his elevated Troponin despite resuscitation, which peaked at $2.6 \mathrm{ng} / \mathrm{mL}$. Echocardiography was performed that demonstrated a preserved ejection fraction, mildly enlarged right ventricle, and a moderately elevated pulmonary artery systolic pressure $(47 \mathrm{~mm} \mathrm{Hg})$, with no evidence of cardiac deformation. Additional diagnostic workup revealed an undetectable TSH $(<0.005 \mu \mathrm{IU} / \mathrm{mL})$ and an elevated free $\mathrm{T} 4(5.54 \mathrm{ng} / \mathrm{dL})$, which were previously noted to be normal one month prior to initiation of Nivolumab/ Ipilimumab. Given his marked PR and ST segment changes, he was given a presumptive diagnosis of immunotherapy-mediated myopericarditis and initiated on $1 \mathrm{mg} / \mathrm{kg}$ Prednisone. Cardiology and Oncology were consulted who noted a low probability of immunotherapy-mediated myopericarditis but agreed with presumptive steroid therapy. He demonstrated clinical improvement on prednisone and was discharged on a two-week taper with plans for outpatient follow up with Oncology.

On follow up two weeks post-discharge, that patient continued to note symptomatic improvement with near resolution of his underlying dyspnea. Repeat echocardiogram demonstrated no significant changes. Laboratory diagnostics demonstrated a down-trending CK (322 units/L) and in discussion with his outpatient Oncologist, steroid therapy was discontinued. Seven days following steroid cessation, the patient experienced recurrence of his shortness of breath and developed associated proximal muscle weakness. Laboratory diagnostics revealed an elevated CK (1,147 units/L) and as a result, he was re-started on steroids and immunotherapy was abandoned.

\section{Discussion}

\section{Mechanisms of $\mathbf{T}$ cell activation: co-stimulation}

In the normal host immune system, T-lymphocytes aid in both surveillance and clearance of neoplastic 
cells. In the context of malignancy, the host immune system generally responds to cancer cells in two ways, either by reacting against tumor-specific antigens (TSA), unique malignancy-associated molecules, or tumor-associated antigens (TAA), molecules that are expressed by cancer cells and normal cells but have divergent post-translational modifications. ${ }^{25,26}$ The antigen presenting cells (APCs), including dendritic cells, natural killer cells, macrophages, and antibody-producing B lymphocytes, sample and process tumorspecific or tumor-associated antigens and present them as TSAs or TAAs to MHC-I or MHC-II molecules on the surface of APCs. T-lymphocytes recognize those antigens (CD4 T-lymphocytes recognize MHC-II and CD8 T-lymphocytes recognize MHC-I, respectively) and attach to them via TCRs. To fully activate T-lymphocytes, however, a second signal is required, mediated by either cytokines or the engagement of costimulatory molecules such as B7.1 (CD80) or B7.2 (CD 86) on APCs. After the two signals co-stimulation, a single T-lymphocyte clone that recognizes a specific tumor antigen is clonally expanded. Compared to helper T-lymphocytes, cytotoxic T-lymphocytes are less reliant on CD28 for activation, but do require signals from other co-stimulatory molecules such as CD70 and CD137. Meanwhile, during the process of T-lymphocyte activation, cytokines (IL-12, IL-6, etc.) induce further differentiation of T-lymphocytes into mature helper T-lymphocytes, Th1 or Th2, each performing a specific task in furthering the immune response. ${ }^{27}$

\section{Immune checkpoints: regulators of the immune system.}

The immune system is a finely tuned temporal balance of pro- and anti-inflammatory signals that allow for appropriately timed inflammatory responses with subsequent resolution. As such, immune system stimulation is balanced by numerous pathways designed to guard against excessive activation and prevent unnecessary damage. Besides the well-known Tregs, which allow immune tolerance and prevention autoimmunity through suppression of CD8 T-lymphocytes, several additional regulatory molecules have been increasingly recognized as having a pivotal role. ${ }^{28-30}$ PD-1 and CTLA-4 (Table 1 ) are two examples of these regulators and are known as immune checkpoint molecules. During the activation of T-lymphocytes, stimulation of CD28 (expressed on T-lymphocytes) by B7 (expressed on APCs) induces T-lymphocytes to produce CTLA-4, a CD28 homolog expressed in low levels on resting T-lymphocytes. ${ }^{27}$ Upon T-lymphocyte activation, CTLA-4 moves from intercellular stores to the T-lymphocyte surface where APC interaction occurs. ${ }^{27,31,32}$ CTLA-4 has a much higher binding affinity for B7, but does not produce a stimulatory signal or even an inhibitory signal, ${ }^{32-35}$ instead competing with CD28 for B7, in effect reducing activating signals to the T-lymphocyte, resulting in anergy of immature/ naïve T-lymphocytes, and dampening the cytotoxic T-lymphocyte immune response. ${ }^{8}$ Moreover, regulatory T-lymphocytes constitutively express CTLA-4, thus potentially contributing to these cells' ability to provide suppressive function. ${ }^{7}$

PD-1 is a glycoprotein receptor expressed on T-lymphocytes, activated B-lymphocytes, natural killer cells, monocytes, and some dendritic cells, while its major ligands (PD-L1 and PD-L2) are found on B-lymphocytes, dendritic cells, macrophages, as well as non-hematopoietic cells, such as vascular endothelial cells, neurons, and certain epithelial cells. ${ }^{36}$ Though PD-L2 has a more potent affinity for PD-1 compared to PD-L1, it is expression repertoire is much more limited, leaving its role in tumor immunity unclear. ${ }^{37}$ If T-lymphocytes experience simultaneous binding of the TCR and PD-1, the interaction of PD-1 with PD-L1 generates signals that prevent phosphorylation of phosphatidylinositol-3-kinase (PI3K) and downstream Akt, ultimately resulting in early termination of TCR signaling and blockade of T-lymphocyte proliferation, cytokine production, cytolytic function, and impaired T-lymphocyte survival. ${ }^{6,38}$ Therefore, PD-1 expression is a hallmark of Tlymphocyte exhaustion, which occurs during chronic infection and cancer, and is characterized by decreased cytokine expression and effector function of mature effector T-lymphocytes. ${ }^{39}$ In addition to the effects on T-lymphocyte, PD-1 signaling has a variety of additional effects - inhibition of macrophages and dendritic cell (DC) responses and blockade of the synthesis and secretion of IL-2, a cytokine crucial in T-lymphocyte proliferation and differentiation. ${ }^{40-43}$

\section{Malignancy and implication of immune checkpoints inhibitors (ICIs)}

Malignant transformation is characterized by the ability to avoid the normal regulatory mechanisms of cell growth, division, and immune-mediated cell death. Indeed, malignant cells are capable of upregulating immune checkpoint proteins - increasing the expression of both PD-L1 as well as CTLA-4. Increased PD-L1 
expression has been observed in multiple malignancies, particularly in melanoma, non-small cell lung cancer (NSCLC), and ovarian cancer. ${ }^{44}$ Preclinical evidence from in vitro and in vivo murine models as well as from clinical trials have shown that malignancy-invading T-lymphocytes can be inhibited by malignancy-related expression of CTLA-4 or PD-1. ${ }^{45-47}$ Ipilimumab, a CTLA-4 inhibitor, was the first treatment demonstrated to prolong overall survival in patients with advanced melanoma across several clinical trials. ${ }^{17,48}$ Following this finding, the application of PD-1 inhibitors in patients with melanoma, NSCLC, or renal cell carcinoma (RCC) also demonstrated significant clinical efficacy, on par with those treated with CTLA-4 inhibitors. ${ }^{49-54}$ Given the distinct mechanisms (Table 1) of CTLA-4 and PD-1 blockade with respect to T-lymphocyte regulation, dual-blockade of both CTLA-4 and PD-1 have been suggested as therapeutic option for advanced malignancies, due to the presumed additive or synergistic, non-overlapping mechanisms. ${ }^{6,55-57}$ Indeed, prior studies have demonstrated that CTLA-4 inhibition induced a proliferative signal mainly in a subset of Tlymphocytes, whereas PD-1 inhibition is associated with cytolysis and natural killer cell function with several preclinical studies and clinical trials demonstrating enhanced antitumor responses using dual ICI therapy as compared to ICI monotherapy, with the potential caveat of increased toxicity. ${ }^{11,13,58-62}$

\section{Immunotherapy-related adverse effects (irAEs)}

Immune checkpoint molecules play a critical role in maintaining immune tolerance and prevent unnecessary damage from excessive activation, involving several different mechanisms. Numerous studies have demonstrated that deficiency or reduced expression of CTLA-4 or PD-1 leads to uncontrolled T-lymphocyte proliferation, autoimmunity, multiorgan failure, and early death. ${ }^{63-67}$ The incidence of irAEs depends on several factors including the type of ICI, dosage, monotherapy or combined therapy, and the cumulative dosage. ${ }^{68}$ According to a meta-analysis synthesized from 22 clinical trials, irAEs cover a broad spectrum across all organ-systems, most commonly manifesting as - dermatologic (eg. vitiligo and self-limited dermatitis), gastrointestinal (eg. Colitis), endocrine (eg. hypothyroidism, hyperthyroidism, type 1 diabetes), hepatitis, and pulmonary (eg. Pneumonitis) manifestations. ${ }^{12,69}$ In histopathological findings from the ICI-mediated irAE vitiligo patients, CD4 and melan-A-specific CD8 T-lymphocytes were found juxtaposed to apoptotic melanocytes. ${ }^{70}$ Similarly, T-lymphocyte infiltration was found in other common irAEs, indicating that ICIs stimulated an immune response directed against normal cells leading to the noted multi-organ irAEs (e.g. colitis, pancreatitis, and endocrinopathies). ${ }^{71,72}$ ICI-mediated myocarditis and pericarditis are less commonly reported, though can be fatal. ${ }^{73,74}$ Previously published data from animal models and case reports have demonstrated the presence of lymphocytic infiltration of the myocardium and pericardium, ${ }^{65,74-76}$ with immunohistochemical analysis revealing the presence of markers for T-lymphocytes (CD3, CD4, CD8, and FOXp3), B-lymphocytes (CD20), macrophages (CD68), PD-L1, and diffuse IgG and complement deposition (C3d, C4d). ${ }^{65,76}$ In a PD-1 deficient mouse model, Nishimura et al. found cardiac-specific autoantibodies. ${ }^{65}$ Taken together, these findings suggest that ICI-mediated myocarditis may result from a common, shared antigen between malignant cells and cardiac myocytes, homologous tumor and myocardial antigens, or dissimilar antigenic targets with shared epitopes. ${ }^{74}$ Further research is warranted to investigate the exact mechanism and variability not only in incidence but the variable patterns of irAEs, even in the context of similar background malignancies in patients treated with ICIs.

The American Society of Clinical Oncology (ASCO) published guidelines in 2018 to aid in the diagnosis and workup of suspected cardiotoxicity secondary to immunotherapy. ${ }^{77}$ These guidelines note that diagnostic evaluation include an ECG, troponin, NT-pro-BNP, and echocardiogram, as well as viral serologies, to exclude alternative causes of cardiac myocyte inflammation. ${ }^{77}$ In a large series, ECG abnormalities were noted in $89 \%$ of cases, troponin elevations were present in $94 \%$ of cases, and echocardiogram was characteristic of a normal LVEF in $51 \%$ of cases. ${ }^{78}$ The severity of myocarditis can furthermore be subclassified into four grades (Table 2 ), with the management tailored to the grade; those with Grade 2 or above require high-dose steroid therapy, whereas those with Grade 4 require aggressive immunosuppression and oftentimes, adjunctive advanced heart failure supportive therapies. ${ }^{77}$ Escudier and colleagues published a descriptive observational analysis of 30 patients with ICI-related cardiotoxicity and noted that, not surprisingly, management ought to be individualized according to cancer status and the presence of toxicity regression. ${ }^{79}$ They furthermore noted that corticosteroid use correlated with improved left ventricular function, and cardiotoxicity did not 
reoccur in those who underwent ICI administration. ${ }^{79}$

Despite the noted deleterious and potentially serious side effects of ICIs, the development and severity of irAEs appeared to be associated with a clinical benefit, according to a recently unpublished study involving 124 patients with advanced sarcoma. ${ }^{80}$ In this study, Rosenbaum and colleagues found that in comparison to those patients that developed an irAE, those patients without irAEs, not only had a decreased response to ICI therapy $(6 \%$ v. $18 \%)$, but also had a lower clinical benefit $(29 \%$ v. $53 \%)$ as well as a decreased median progression-free survival (10.6 v. 16.4 weeks) ${ }^{80}$ Furthermore, when comparing patients with lower grade irAEs (Grade 1-2) or no irAEs to those patients with higher grade irAEs (grade 3-4), the patients in the higher grade irAE group were noted to have a significantly increased response rate $(33 \% \mathrm{v} .15 \% \mathrm{v} .6 \%)$, a higher durable clinical response (67\% v. $50 \%$ v. $29 \%$ ), and a longer median progression-free survival (22.6 v. 15 v. 10.6 weeks). ${ }^{80}$

Our patient presented with a constellation of symptoms; with. his initial shortness of breath aptly and quickly attributed to an irAE in the setting of his ICI therapy for his renal cell cancer, and he was promptly initiated on high dose steroids. His presenting laboratory diagnostics demonstrated mild transaminitis, hyperthyroidism, and an elevated CK and troponin. Diagnostic imaging demonstrated the presence of a persistent mass abutting the SVC, however physical examination demonstrated no evidence of SVC syndrome and IV hydration failed to improve his symptomatology. Furthermore, while he had a prior history of radiation, the temporal relationship between the onset of his immunotherapy and symptoms made radiation-induced injury less likely. ${ }^{81}$ As such it appears that he presented with a mild systemic inflammatory response secondary to ICI therapy, which subsequently responded to steroid therapy. He was ultimately diagnosed with Grade 2 myocarditis given his mild symptoms and lab abnormalities. Though the exact pathophysiological mechanism remains unknown, it is likely that in this case ICI therapy resulted in a down-regulation of the normal immune-regulatory signaling mediated by CTLA-4 and PD-1, with resultant cross-reactivity of malignancyassociated antigens with antigens on healthy myocytes. In the ideal environment, regulatory T-lymphocytes would ordinarily recognize the healthy cell antigen and suppress the immune response, however in the setting of ICI therapy, these normal regulatory events are abrogated. As the patient's symptoms returned with cessation of steroids, we suspect that the patient's presentation was driven by the development of an autoimmune response with associated autoantibody production. Alternatively, his presentation may also be explained by IgG and complement deposition, as suggested in Nishimura's study, which has been suggested to play a role in the recurrence of symptoms. ${ }^{65}$ This would place our patient in a different subset of patient's who develop irAEs, as this implicates involvement of the adaptive immune system and development of memory and effector T-lymphocytes.

Our patient also presented with thyrotoxicosis. Immune-related thyroiditis usually manifests as an early onset of thyrotoxicosis, which is largely asymptomatic, followed by rapid transition to hypothyroidism requiring long-term levothyroxine substitution. The incidence of immune-related thyroiditis is notably higher and occurs with increased rapidity in those treated with a combination of ICI, warranting frequent monitoring of thyroid function tests. Our patient had routine follow up with his oncologist for close monitoring of thyroid function tests and subsequent initiation of levothyroxine supplementation.

\section{Conclusions/Future Directions}

It is important to have a high clinical suspicion for irAEs in those undergoing treatment with novel immunotherapies for their malignancies. These therapies are promising and provide hope for an improved length of survival and quality of life in those with otherwise poor outcomes, however their associated toxicities, which manifest as irAEs may be fatal. Immunotherapy related cardiac toxicity is an important albeit rare sequalae of immune checkpoint inhibitor therapy. Further studies are needed to evaluate the risks and benefits of staggered induction therapy, as has been studied in melanoma, as well as dosing manipulation to reduce the effects of combined drug therapy. ${ }^{82}$ As more cases of irAEs are reported, it will be important not only to determine the underlying genetic determinants that predispose to irAEs, in order to specific tailor therapy to the patients individual genetic predisposition, but also to longitudinally follow these patients to determine resolution of these presentations and their effects on outcomes. 


\section{Funding: None}

Disclosure statement: The authors whose names are listed immediately below certify that they have NO affiliations with or involvement in any organization or entity with any financial interest (such as honoraria; educational grants; participation in speakers' bureaus; membership, employment, consultancies, stock ownership, or other equity interest; and expert testimony or patient-licensing arrangements), or non-financial interest (such as personal or professional relationships, affiliations, knowledge or beliefs) in the subject matter or materials discussed in this manuscript.

Institutional Review Board approval was not sought as this represents a single case and did not involve human subject research. Ethics approval was not sought as care provided was considered to be standard of care with no ethical dilemmas noted.

\section{References}

1. Sun Z, Fourcade J, Pagliano O, et al. Interleukin-10 and programmed death-1 cooperate to regulate tumor antigen-specific CD8+ T cells in melanoma patients. In: AACR; 2015.

2. Jana D, Sarkar DK, Ganguly S, et al. Role of cyclooxygenase 2 (COX-2) in prognosis of breast cancer. Indian journal of surgical oncology. 2014;5(1):59-65.

3. Wu C-T, Chang Y-H, Lin W-Y, Chen W-C, Chen M-F. TGF beta1 expression correlates with survival and tumor aggressiveness of prostate cancer.Annals of surgical oncology. 2015;22(3):1587-1593.

4. Yang $\mathrm{C}, \mathrm{He} \mathrm{L}, \mathrm{He} \mathrm{P}$, et al. Increased drug resistance in breast cancer by tumor-associated macrophages through IL-10/STAT3/bcl-2 signaling pathway. Medical oncology. 2015;32(2):14.

5. Masuda K, Hiraki A, Fujii N, et al. Loss or down-regulation of HLA class I expression at the allelic level in freshly isolated leukemic blasts. Cancer Science. 2007;98(1):102-108.

6. Parry RV, Chemnitz JM, Frauwirth KA, et al. CTLA-4 and PD-1 receptors inhibit T-cell activation by distinct mechanisms. Molecular and cellular biology. 2005;25(21):9543-9553.

7. Walker LS. Treg and CTLA-4: two intertwining pathways to immune tolerance. Journal of autoimmunity. 2013;45:49-57.

8. Nirschl CJ, Drake CG. Molecular pathways: coexpression of immune checkpoint molecules: signaling pathways and implications for cancer immunotherapy. Clinical cancer research. 2013;19(18):4917-4924.

9. Chen L, Flies DB. Molecular mechanisms of T cell co-stimulation and co-inhibition. Nature Reviews Immunology. 2013;13(4):227-242.

10. Huse M. The T-cell-receptor signaling network. J Cell Sci.2009;122(9):1269-1273.

11. Curran MA, Montalvo W, Yagita H, Allison JP. PD-1 and CTLA-4 combination blockade expands infiltrating T cells and reduces regulatory T and myeloid cells within B16 melanoma tumors. Proc Natl Acad Sci U S A. 2010;107(9):4275-4280.

12. Larkin J, Hodi FS, Wolchok JD. Combined Nivolumab and Ipilimumab or Monotherapy in Untreated Melanoma. N Engl J Med.2015;373(13):1270-1271.

13. Postow MA, Chesney J, Pavlick AC, et al. Nivolumab and ipilimumab versus ipilimumab in untreated melanoma. N Engl J Med.2015;372(21):2006-2017.

14. Hammers HJ, Plimack ER, Infante JR, et al. Safety and efficacy of nivolumab in combination with ipilimumab in metastatic renal cell carcinoma: the CheckMate 016 study. J Clin Oncol.2017;35(34):38513858.

15. Motzer RJ, Escudier B, McDermott DF, et al. Nivolumab versus everolimus in advanced renal-cell carcinoma. New England Journal of Medicine. 2015;373(19):1803-1813. 
16. Overman MJ, McDermott R, Leach JL, et al. Nivolumab in patients with metastatic DNA mismatch repair-deficient or microsatellite instability-high colorectal cancer (CheckMate 142): an open-label, multicentre, phase 2 study. The Lancet Oncology.2017;18(9):1182-1191.

17. Hodi FS, O'Day SJ, McDermott DF, et al. Improved survival with ipilimumab in patients with metastatic melanoma. N Engl J Med.2010;363(8):711-723.

18. Brahmer J, Reckamp KL, Baas P, et al. Nivolumab versus Docetaxel in Advanced Squamous-Cell NonSmall-Cell Lung Cancer. N Engl J Med.2015;373(2):123-135.

19. Prior LM, Harrold E, O'Leary CG, et al. Toxicities in immunotherapy: Can they predict response? In: American Society of Clinical Oncology; 2016.

20. Varricchi G, Marone G, Mercurio V, Galdiero MR, Bonaduce D, Tocchetti CG. Immune Checkpoint Inhibitors and Cardiac Toxicity: An Emerging Issue. Curr Med Chem. 2018;25(11):1327-1339.

21. Ganatra S, Neilan TG. Immune Checkpoint Inhibitor-Associated Myocarditis. Oncologist. 2018;23(8):879886.

22. Atallah-Yunes SA, Kadado AJ, Kaufman GP, Hernandez-Montfort J. Immune checkpoint inhibitor therapy and myocarditis: a systematic review of reported cases. J Cancer Res Clin Oncol.2019;145(6):1527-1557.

23. Maughan BL, Bailey E, Gill DM, Agarwal N. Incidence of immune-related adverse events with program death receptor-1-and program death receptor-1 ligand-directed therapies in genitourinary cancers.Frontiers in oncology. 2017;7:56.

24. Palaskas N, Lopez-Mattei J, Durand JB, Iliescu C, Deswal A. Immune Checkpoint Inhibitor Myocarditis: Pathophysiological Characteristics, Diagnosis, and Treatment. Journal of the American Heart Association. 2020;9(2):e013757.

25. Graziano DF, Finn OJ. Tumor antigens and tumor antigen discovery. In: Tumor Immunology and Cancer Vaccines. Springer; 2005:89-111.

26. Srivastava PK, Old LJ. Individually distinct transplantation antigens of chemically induced mouse tumors. Immunology today.1988;9(3):78-83.

27. Smith-Garvin JE, Koretzky GA, Jordan MS. T Cell Activation.Annual Review of Immunology. 2009;27(1):591-619.

28. Belkaid Y. Regulatory $\mathrm{T}$ cells and infection: a dangerous necessity.Nature Reviews Immunology. 2007;7(11):875-888.

29. Veiga-Parga T, Sehrawat S, Rouse BT. Role of regulatory T cells during virus infection. Immunological reviews.2013;255(1):182-196.

30. Nishikawa H, Sakaguchi S. Regulatory $\mathrm{T}$ cells in tumor immunity.International journal of cancer. 2010;127(4):759-767.

31. Morse MA. Technology evaluation: ipilimumab, Medarex/Bristol-Myers Squibb. Current opinion in molecular therapeutics.2005;7(6):588-597.

32. Chambers CA, Kuhns MS, Egen JG, Allison JP. CTLA-4-mediated inhibition in regulation of T cell responses: mechanisms and manipulation in tumor immunotherapy. Annual review of immunology.2001;19(1):565-594.

33. Collins AV, Brodie DW, Gilbert RJ, et al. The interaction properties of costimulatory molecules revisited. Immunity.2002;17(2):201-210.

34. Fallarino F, Fields PE, Gajewski TF. B7-1 engagement of cytotoxic T lymphocyte antigen 4 inhibits T cell activation in the absence of CD28. The Journal of experimental medicine. 1998;188(1):205-210. 
35. Masteller EL, Chuang E, Mullen AC, Reiner SL, Thompson CB. Structural analysis of CTLA-4 function in vivo. The Journal of Immunology. 2000;164(10):5319-5327.

36. Francisco LM, Sage PT, Sharpe AH. The PD-1 pathway in tolerance and autoimmunity. Immunological reviews. 2010;236(1):219-242.

37. Yang H, Zhou X, Sun L, Mao Y. Correlation Between PD-L2 Expression and Clinical Outcome in Solid Cancer Patients: A Meta-Analysis.Frontiers in Oncology. 2019;9(47).

38. Bennett F, Luxenberg D, Ling V, et al. Program death-1 engagement upon TCR activation has distinct effects on costimulation and cytokine-driven proliferation: attenuation of ICOS, IL-4, and IL-21, but not CD28, IL-7, and IL-15 responses. The Journal of Immunology.2003;170(2):711-718.

39. Wherry EJ. T cell exhaustion. Nature immunology.2011;12(6):492-499.

40. Riley JL. PD-1 signaling in primary T cells. Immunological reviews. 2009;229(1):114-125.

41. Yao S, Wang S, Zhu Y, et al. PD-1 on dendritic cells impedes innate immunity against bacterial infection. Blood, The Journal of the American Society of Hematology. 2009;113(23):5811-5818.

42. Huang X, Venet F, Wang YL, et al. PD-1 expression by macrophages plays a pathologic role in altering microbial clearance and the innate inflammatory response to sepsis. Proceedings of the National Academy of Sciences. 2009;106(15):6303-6308.

43. Carter LL, Fouser LA, Jussif J, et al. PD-1: PD-L inhibitory pathway affects both CD4+ and CD8+ T cells and is overcome by IL-2.European journal of immunology. 2002;32(3):634-643.

44. Taube JM, Klein A, Brahmer JR, et al. Association of PD-1, PD-1 Ligands, and Other Features of the Tumor Immune Microenvironment with Response to Anti-PD-1 Therapy. Clinical Cancer Research.2014;20(19):5064-5074.

45. Loi S, Dushyanthen S, Beavis PA, et al. RAS/MAPK activation is associated with reduced tumorinfiltrating lymphocytes in triple-negative breast cancer: therapeutic cooperation between MEK and PD1/PD-L1 immune checkpoint inhibitors. Clinical Cancer Research. 2016;22(6):1499-1509.

46. Peggs KS, Quezada SA, Allison JP. Cell intrinsic mechanisms of T-cell inhibition and application to cancer therapy. Immunological reviews. 2008;224(1):141-165.

47. Okazaki T, Honjo T. PD-1 and PD-1 ligands: from discovery to clinical application. International immunology.2007;19(7):813-824.

48. Schadendorf D, Hodi FS, Robert C, et al. Pooled analysis of long-term survival data from phase II and phase III trials of ipilimumab in unresectable or metastatic melanoma. Journal of clinical oncology. 2015;33(17):1889.

49. Herbst RS, Soria JC, Kowanetz M, et al. Predictive correlates of response to the anti-PD-L1 antibody MPDL3280A in cancer patients. Nature. 2014;515(7528):563-567.

50. Farolfi A, Ridolfi L, Guidoboni M, et al. Ipilimumab in advanced melanoma: reports of long-lasting responses. Melanoma research.2012;22(3):263-270.

51. Garon EB, Rizvi NA, Hui R, et al. Pembrolizumab for the treatment of non-small-cell lung cancer. New England Journal of Medicine.2015;372(21):2018-2028.

52. Gettinger SN, Horn L, Gandhi L, et al. Overall survival and long-term safety of nivolumab (antiprogrammed death 1 antibody, BMS-936558, ONO-4538) in patients with previously treated advanced nonsmall-cell lung cancer. Journal of clinical oncology.2015;33(18):2004. 
53. McDermott DF, Drake CG, Sznol M, et al. Survival, durable response, and long-term safety in patients with previously treated advanced renal cell carcinoma receiving nivolumab. Journal of clinical oncology.2015;33(18):2013.

54. Topalian SL, Sznol M, McDermott DF, et al. Survival, durable tumor remission, and long-term safety in patients with advanced melanoma receiving nivolumab. Journal of clinical oncology.2014;32(10):1020.

55. Fife BT, Bluestone JA. Control of peripheral T-cell tolerance and autoimmunity via the CTLA-4 and PD-1 pathways. Immunological reviews. 2008;224(1):166-182.

56. Keir ME, Butte MJ, Freeman GJ, Sharpe AH. PD-1 and its ligands in tolerance and immunity. Annu Rev Immunol. 2008;26:677-704.

57. Wing K, Onishi Y, Prieto-Martin P, et al. CTLA-4 control over Foxp3+ regulatory T cell function. Science. 2008;322(5899):271-275.

58. Goldrath AW, Bevan MJ. Selecting and maintaining a diverse T-cell repertoire. Nature. $1999 ; 402(6759): 255-262$.

59. Buchbinder E, Hodi FS. Cytotoxic T lymphocyte antigen-4 and immune checkpoint blockade. J Clin Invest. 2015;125(9):3377-3383.

60. Das R, Verma R, Sznol M, et al. Combination therapy with anti-CTLA-4 and anti-PD-1 leads to distinct immunologic changes in vivo. The Journal of Immunology. 2015;194(3):950-959.

61. Selby M, Engelhardt J, Lu L-S, et al. Antitumor activity of concurrent blockade of immune checkpoint molecules CTLA-4 and PD-1 in preclinical models. In: American Society of Clinical Oncology; 2013.

62. Wolchok JD, Kluger H, Callahan MK, et al. Nivolumab plus ipilimumab in advanced melanoma. $N$ Engl J Med. 2013;369:122-133.

63. Tivol EA, Borriello F, Schweitzer AN, Lynch WP, Bluestone JA, Sharpe AH. Loss of CTLA-4 leads to massive lymphoproliferation and fatal multiorgan tissue destruction, revealing a critical negative regulatory role of CTLA-4. Immunity. 1995;3(5):541-547.

64. Waterhouse P, Penninger JM, Timms E, et al. Lymphoproliferative disorders with early lethality in mice deficient in Ctla-4.Science. 1995;270(5238):985-988.

65. Nishimura H, Okazaki T, Tanaka Y, et al. Autoimmune dilated cardiomyopathy in PD-1 receptordeficient mice. Science.2001;291(5502):319-322.

66. Ansari MJI, Salama AD, Chitnis T, et al. The programmed death-1 (PD-1) pathway regulates autoimmune diabetes in nonobese diabetic (NOD) mice. The Journal of experimental medicine. 2003;198(1):63-69.

67. Kristiansen O, Larsen Z, Pociot F. CTLA-4 in autoimmune diseases-a general susceptibility gene to autoimmunity? Genes $\mathcal{G}$ Immunity.2000;1(3):170-184.

68. Tocut M, Brenner R, Zandman-Goddard G. Autoimmune phenomena and disease in cancer patients treated with immune checkpoint inhibitors.Autoimmunity reviews. 2018;17(6):610-616.

69. Menzies AM, Johnson D, Ramanujam S, et al. Anti-PD-1 therapy in patients with advanced melanoma and preexisting autoimmune disorders or major toxicity with ipilimumab. Annals of Oncology.2017;28(2):368-376.

70. Downey SG, Klapper JA, Smith FO, et al. Prognostic factors related to clinical response in patients with metastatic melanoma treated by CTL-associated antigen-4 blockade. Clinical Cancer Research.2007;13(22):6681-6688.

71. Weber JS, Kahler KC, Hauschild A. Management of immune-related adverse events and kinetics of response with ipilimumab. Journal of Clinical Oncology. 2012;30(21):2691-2697. 
72. Wolchok JD, Neyns B, Linette G, et al. Ipilimumab monotherapy in patients with pretreated advanced melanoma: a randomised, double-blind, multicentre, phase 2, dose-ranging study. The lancet oncology.2010;11(2):155-164.

73. Roth ME, Muluneh B, Jensen BC, Madamanchi C, Lee CB. Left ventricular dysfunction after treatment with ipilimumab for metastatic melanoma. American journal of therapeutics.2016;23(6):e1925-e1928.

74. Johnson DB, Balko JM, Compton ML, et al. Fulminant Myocarditis with Combination Immune Checkpoint Blockade. N Engl J Med.2016;375(18):1749-1755.

75. Ji C, Roy MD, Golas J, et al. Myocarditis in cynomolgus monkeys following treatment with immune checkpoint inhibitors. Clinical Cancer Research. 2019;25(15):4735-4748.

76. Balanescu DV, Donisan T, Palaskas N, et al. Immunomodulatory treatment of immune checkpoint inhibitor-induced myocarditis: Pathway toward precision-based therapy. Cardiovasc Pathol.2020;47:107211.

77. Brahmer JR, Lacchetti C, Schneider BJ, et al. Management of immune-related adverse events in patients treated with immune checkpoint inhibitor therapy: American Society of Clinical Oncology Clinical Practice Guideline. Journal of clinical oncology: official journal of the American Society of Clinical Oncology. 2018;36(17):1714.

78. Mahmood SS, Fradley MG, Cohen JV, et al. Myocarditis in Patients Treated With Immune Checkpoint Inhibitors. J Am Coll Cardiol.2018;71(16):1755-1764.

79. Escudier M, Cautela J, Malissen N, et al. Clinical Features, Management, and Outcomes of Immune Checkpoint Inhibitor-Related Cardiotoxicity. In: Circulation. Vol 136. United States2017:2085-2087.

80. Rosenbaum E, Seier K, Kelly CM, et al. Association of immune-related adverse events (irAEs) with improved clinical outcome in sarcoma patients treated with immune checkpoint blockade (ICB). 2020 ASCO Virtual Scientific Program; 2020.

81. Heidenreich PA, Kapoor JR. Radiation induced heart disease.Heart. 2009;95(3):252-258.

82. Nakamura K, Okuyama R. Immunotherapy for advanced melanoma: Current knowledge and future directions. Journal of dermatological science. 2016;83(2):87-94.

83. Linsley PS, Bradshaw J, Greene J, Peach R, Bennett KL, Mittler RS. Intracellular trafficking of CTLA-4 and focal localization towards sites of TCR engagement. Immunity. 1996;4(6):535-543.

84. Egen JG, Allison JP. Cytotoxic T lymphocyte antigen-4 accumulation in the immunological synapse is regulated by TCR signal strength.Immunity. 2002;16(1):23-35.

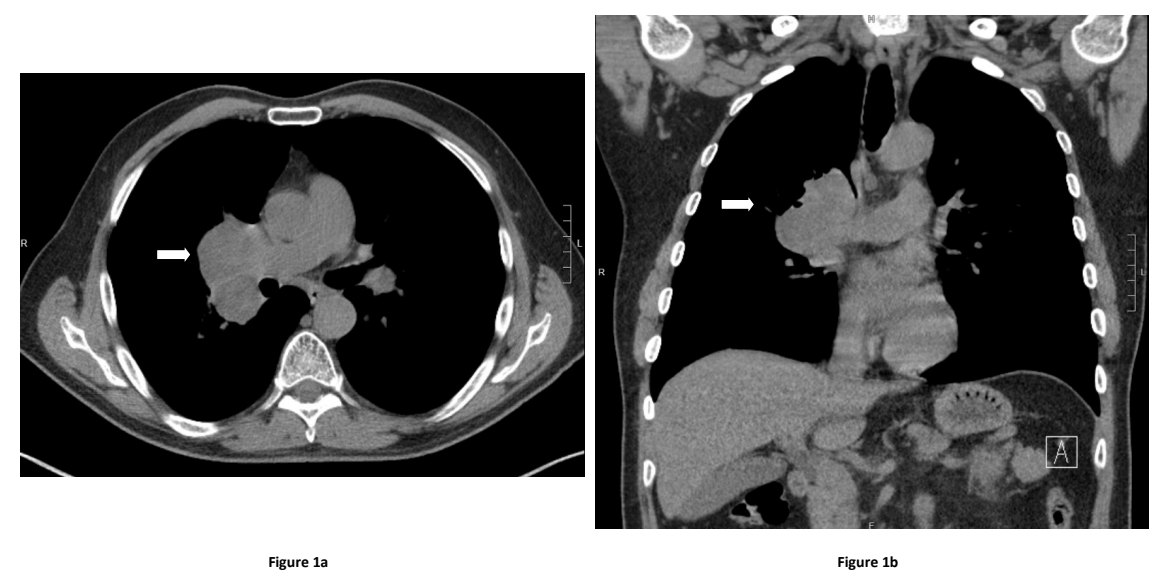



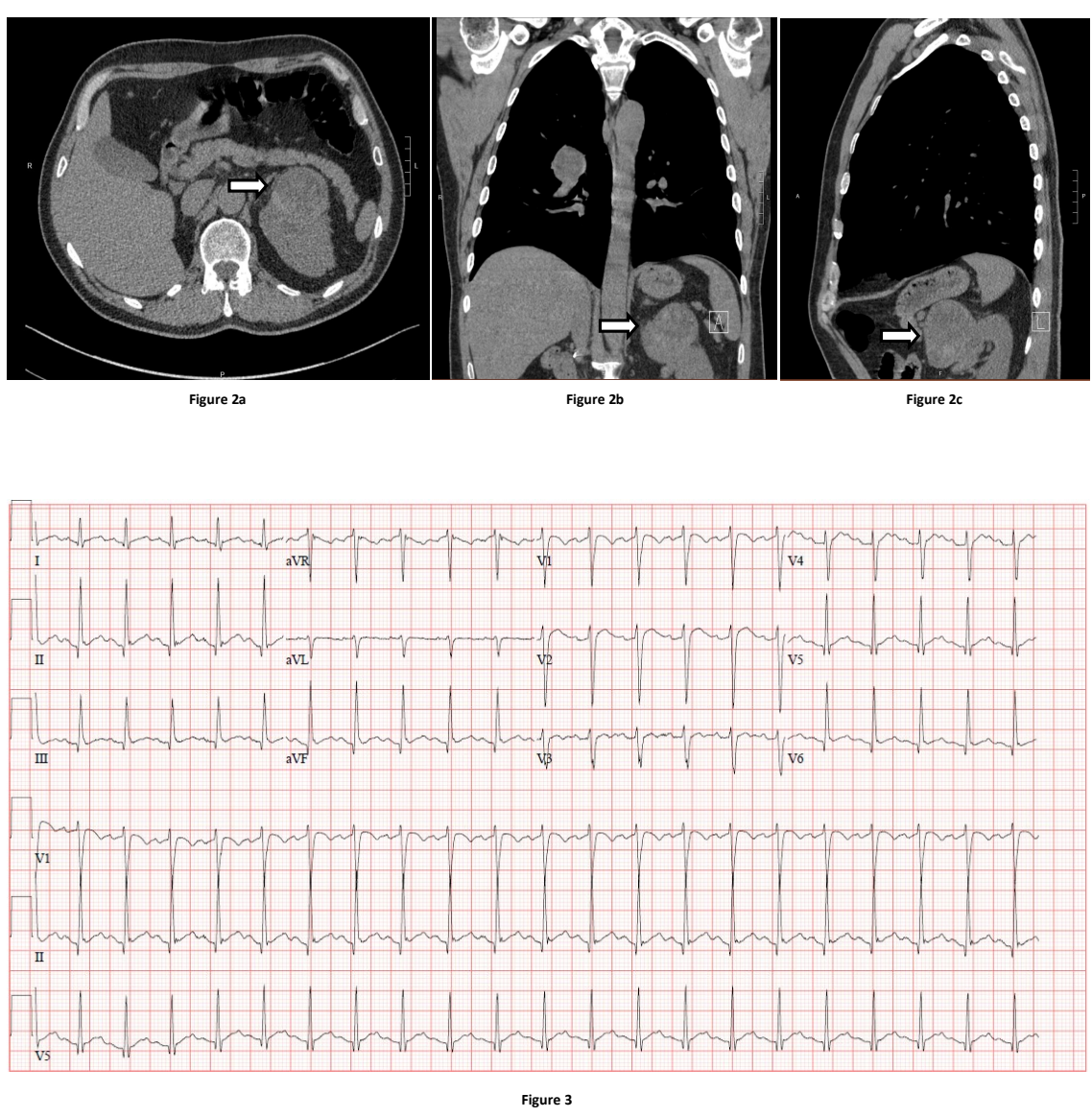